\title{
Ventricular fibrillation and reinfarction in pregnancy
}

\author{
M. O'Donnell, J. Meecham, S.R. Tosson and S. Ward
}

Arrowe Park Hospital, Upton, Wirral, Merseyside, L49 5PE, UK.

\begin{abstract}
Summary: This is the first reported case of a woman suffering a potentially fatal dysrhythmia and reinfarction during the same pregnancy and surviving to produce a healthy, live infant.
\end{abstract}

\section{Introduction}

We report a woman suffering ventricular fibrillation due to acute myocardial infarction in the middle trimester, reinfarction later in pregnancy and surviving to produce a live healthy infant.

\section{Case report}

A 39 year old para $5+5$ housewife who smoked 15 cigarettes a day and weighed $85 \mathrm{~kg}$ was booked for ante-natal care at 16 weeks gestation. She had no significant medical or family history. At 18 weeks gestation she presented complaining of retrosternal discomfort and vomiting for one hour. Her pulse was 84 beats/minute and blood pressure $130 / 70 \mathrm{~mm} \mathrm{Hg}$, as at her ante-natal clinic visit.

While in the Casualty Department she collapsed and was found to be in ventricular fibrillation. She was successfully cardioverted with one shock of 320 Joules and transferred to the coronary care unit. Her blood pressure was $90 / 80 \mathrm{~mm} \mathrm{Hg}$. The fetal heart rate was 140 beats/minute and regular. The electrocardiogram showed evidence of a fresh anteroseptal transmural infarction and this was confirmed by an elevation of the cardiac enzymes. A random blood sugar was normal. Fasting lipids 12 weeks post infarct were normal.

The subsequent course of the pregnancy was uneventful until 33 weeks gestation when she was readmitted as an emergency with a further episode of chest pain. Her blood pressure was $75 / 50 \mathrm{~mm} \mathrm{Hg}$ and pulse rate 110 beats/minute. The electrocardiogram showed fresh changes of an acute anterior myocardial infarction and cardiac enzymes were again elevated. She was re-admitted to the coronary care unit but took

Correspondence: M. O'Donnell, M.B., Ch.B., M.R.C.P. (UK), Research Fellow, Department of Medicine, East Birmingham Hospital, Bordesley Green East, Birmingham B9 5ST, U.K.

Accepted: 30 July 1987 her own discharge against medical advice after four days.

The remainder of the pregnancy was marked by increasing breathlessness without overt cardiac failure. Fetal growth was normal.

She was readmitted at term. Labour was induced with prostaglandin E3 pessaries and progressed satisfactorily until the fetal cardiotocograph showed decelerative changes in association with maternal chest pain. The pain was rapidly relieved by sublingual glyceryl trinitrate and the decelerative changes disappeared following this. A live male infant $(3.08 \mathrm{~kg})$ was delivered with Wrigley's forceps. Apgar score was 7 at one minute and 9 at three minutes.

The child had passed all his developmental milestones at twelve months. The patient remains severely breathless on exertion having an exercise tolerance of only twenty yards. The most recent electrocardiogram shows the previous infarctions but no other ischaemic changes. An echocardiograph recorded several months post-delivery showed a dilated left ventricle with an akinetic septum and reduced posterior wall movement. A resting thallium scan confirms the dilatation of the left ventricle and shows decreased uptake by the myocardium and abnormal uptake in the lungs.

\section{Discussion}

Myocardial infarction in pregnancy is a rare condition. It was first reported by Katz in 1922 and has an estimated incidence of 1 in 10,000 deliveries. ${ }^{2}$ There are only 68 well documented cases reported in the literature and there are only four cases of reinfarction.

The disease carries a poor prognosis with an overall maternal mortality of $28 \%$. Mortality ranges from nil in the first trimester to $40 \%$ in the second, $21 \%$ in the third and $50 \%$ in the puerperium. ${ }^{2}$ The mortality also varies with age. Surprisingly women of 35 years or older have a better prognosis than those who are 
younger ( $34 \%$ mortality $v s 43 \%){ }^{3}$

From the 68 cases there are five cases of successful cardioversion with delivery of a live infant in four. ${ }^{3-7}$ Our report is we believe the first report of a woman surviving cardioversion from ventricular fibrillation and a second myocardial infarction in pregnancy to produce a live infant.

The reasons for myocardial infarction and its variable mortality in pregnancy are unknown. Our patient was at risk because she was an obese smoker. However, she had no antecedent heart trouble and no other known risk factors. There have been several reports of myocardial infarction with normal coronary arteriograms and it was suggested that these were due to coronary artery spasm provoked by angiotensin stimulated by uterine renin release. ${ }^{8}$ All of these reports were of patients presenting in the third trimester and the proposed initiating factor (decreased uterine blood flow induced by lying supine) would not apply in this patient's first presentation although it may have had a role in the second.

\section{References}

1. Katz, H. About the sudden natural death in pregnancy: during delivery and puerperium. Archiv Gynaekol 1922, 115: 283.

2. Ginz, B. Myocardial infarction in pregnancy. J Obstet Gynaec Brit Comm 1970, 77: 610-615.

3. Hankins, G.D.V., Wendel, G.R., Leveno, K.J. \& Stoneham, J. Myocardial infarction in pregnancy: a review. Obstet Gynecol 1985, 65: 139-146.

4. Curry, J.J. \& Quintana, F.J. Myocardial infarction with ventricular fibrillation during pregnancy treated by direct current defibrillation with fetal survival. Chest, 1970, 58: 82-84.

5. Cortis, B.S., Lee, S.S. \& Bacalla, M. Acute myocardial infarction and ventricular infarction during pregnancy. IMJ 1981, 160(3): 170-174.

6. Cohen, W.R., Steinman, T., Patsner, B., Snyder, D., Sat-
This patient's second infarction occurred in the third trimester and this accords well with the four previous reports of reinfarction in pregnancy. ${ }^{9} \mathrm{Re}$ infarction seems to have a particularly high mortality if it occurs during labour and our patient (who had chest pain at this stage) was fortunate to survive.

The management of labour is problematical. Induction with oxytocin is theoretically disadvantageous in this situation because of fluid retention and effects on myocardial function. Animal experiments have shown that prostaglandins of the $\mathrm{E}$ series have some cardiodilator activity so there may be some rationale for their use as labour inducers in this type of case. ${ }^{10}$

It appears that vaginal delivery is safer than Caesarian section unless there is a specific obstetric indication for the latter.$^{2,3}$ Labour must not be prolonged because of the strain on the myocardium and it is probably wise to assist the second stage.

Our patient has been left with a greatly reduced cardiac reserve and must avoid further pregnancy. She has refused any further cardiological investigation.

nicz, P. \& Monroy, P. Acute myocardial infarction in a pregnant woman at term. JAMA 1983, 250: 2179-2181

7. Stokes, I.M., Evans, J. \& Stone, M. Myocardial infarc tion and cardiac arrest in the second trimester followed by assisted vaginal delivery under epidural analgesia at? 38 weeks gestation. A case report. Br J Obstet Gynaecol 1984, 91: 197-198.

8. Sasse, L., Wagner, R. \& Murray, F.E. Transmural myocardial infarction during pregnancy. Am J Cardiol 1975, 35: 448-452.

9. Cullned, I. Cardioversion during pregnancy. A case report. Acta Med Scand 1983, 214: 169-172.

10. Page, E.W. On the pathogenesis of pre-eclampsia and eclampsia. J Obset Gynaec Brit Comm 1972, 79: 883894. 